\title{
Liquen plano oral (II). Mecanismos apoptóticos y posible malignización
}

\author{
Bascones-Ilundain $C^{*}$, González Moles MA**, Campo-Trapero J***, \\ Bascones-Martínez A****
}

\section{RESUMEN}

Se presenta un estudio histopatológico realizado con una muestra de 52 pacientes con liquen plano oral evaluados en la Universidad Complutense de Madrid con el objetivo de valorar los fenómenos apoptóticos y su posible vinculación con la malignización de las lesiones.

Palabras clave: Liquen plano oral, apoptosis, malignización, caspasas, bax, TUNEL.

\section{SUMMARY}

A histopathological study is carried out on 52 patients with oral lichen planus at the Faculty of Dentistry of Madrid, with the aim to assess apoptotic phenomena and their possible relationship with the malignization of the lesions.

Key words: Oral lichen planus, apoptosis, malignization, caspases, bax, TUNEL.

\section{Aceptado para publicación:}

* Doctora en Odontología y Master por la Universidad Complutense de Madrid.

** Profesor Titular de Medicina Bucal. Facultad de Odontología. Universidad de Granada.

*** Profesor contratado doctor. Facultad de Odontología. Universidad Complutense de Madrid.

**** Catedrático de Medicina Bucal y Periodoncia. Facultad de Odontología. Universidad Complutense de Madrid.

Bascones-llundain C, González Moles MA, Campo-Trapero J, Bascones-Martínez A. Liquen plano oral (II). Mecanismos apoptóticos y posible malignización. Av. Odontoestomatol 2006; 22-1: 21-31.

\section{INTRODUCCIÓN}

La evolución del LPO es crónica con periodos de exacerbación y remisión habiendo mucha controversia sobre la posibilidad de malignización en función del grado de displasia existente. Las fases atróficaserosivas son las que tienen más posibilidades de malignización.
Desde el punto de vista histopatológico en la mucosa oral afectada por el LP se pueden presentar alteraciones diferentes como hiperqueratosis (orto o paraqueratósica), acantosis, granulosis, espongiosis, cuerpos coloides, exocitosis linfocitaria y atrofia epitelial. Aunque los hechos esenciales de la enfermedad están constituidos por dos fenómenos fundamentales, la degeneración vacuolizante de la capa 
basal del epitelio y el infiltrado inflamatorio subyacente en banda de tipo intenso.

Para algunos autores, la degeneración de la capa basal es un signo histomorfológico de apoptosis celular, que debería definirse según (1), por la presencia de condensación cromática en la periferia del núcleo y/o citoplasmática, picnosis nuclear, y desintegración nucleolar.

La apoptosis es un fenómeno celular de enorme trascendencia por el que las células mueren tomando parte activa en su propia eliminación. Se denomina muerte celular programada, produciéndose de manera habitual en los organismos pluricelulares, y tiene como misión eliminar células dañadas o inútiles, sin que esto tenga repercusión sobre las células vecinas. La apoptosis es, por tanto, un evento esencial dentro de los fenómenos que acontecen en el ciclo celular $(2,3)$.

Es un proceso fisiológico que se activa mediante la acción de proteasas específicas y de endonucleasas. Se produce la formación y secreción de vesículas de la membrana, la condensación y fragmentación de la cromatina y formación de cuerpos apoptóticos. En la fase inicial, la célula pierde el contacto con las células que le rodean. Tiene lugar la formación de protuberancias en la membrana plasmática y la cromatina se condensa y fragmenta, permaneciendo la envoltura nuclear. El volumen citoplasmático disminuye por la pérdida de agua y por la condensación de las proteínas. En una segunda etapa se fragmentan la membrana plasmática, apareciendo los cuerpos apoptóticos que contienen en su interior material nuclear y citoplasmático. Finalmente en la tercera etapa se produce la fagocitosis de los cuerpos apoptóticos por parte de las macrófagos y su degradación. (Figura 1).

Estas fases de apoptosis se activan por medio de una serie de efectores moleculares entre los que destaca la actividad de las caspasas como componentes fundamentales de la maquinaria apoptótica de los mamíferos. Se trata de proteasas del tipo cisteinproteasas de las que resulta especialmente importante la Caspasa-3 por romper y activar otras caspasas que tienen dianas relevantes en el citoplasma y el núcleo, y es responsable de la proteólisis. (4).

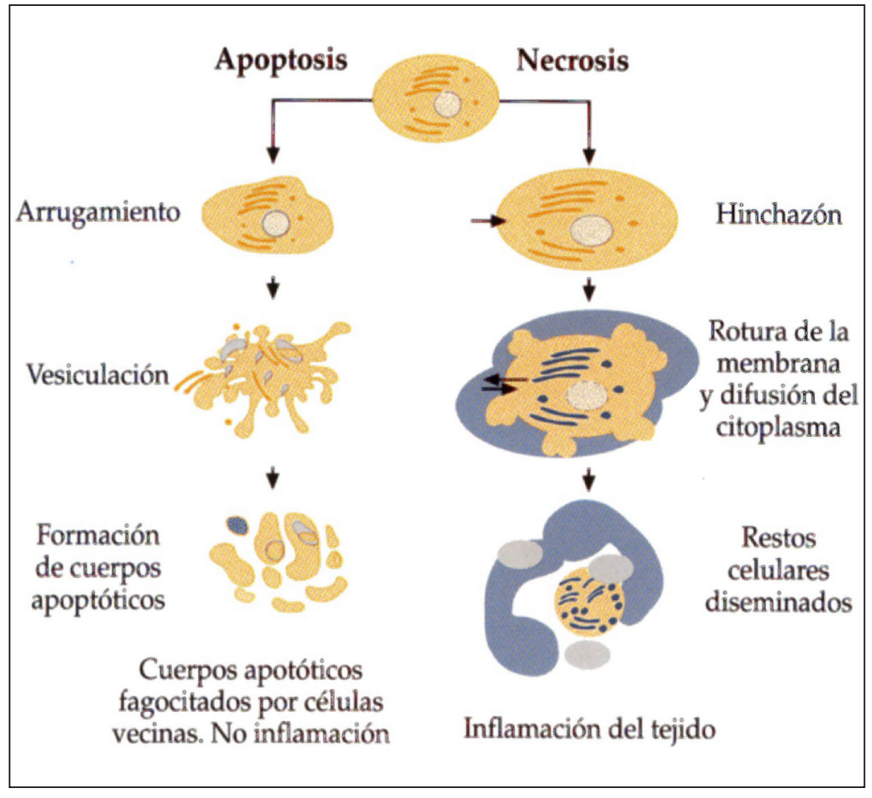

Figura 1. Principales cambios morfológicos de los procesos de muerte celular necrótica y apoptótica (Diéz y cols. 1999).

Bax es considerado otro inductor de apoptosis, que pertenece a la familia $\mathrm{Bcl}-2$, la forma activa inductora de apoptosis es Bax- $\alpha$ (5).

La técnica TUNEL (transfer uridin nick-end Labelling). Se detecta la fragmentación del ADN en escalera en los núcleos apoptóticos, se produce de forma rápida y sencilla y ha demostrado su eficacia en la detección de apoptosis en células cultivadas (6).

Otros fenómenos moleculares importantes que acontecen en la regulación del ciclo celular son la detención del ciclo celular y la senescencia celular. Mediante la detención del ciclo celular (se permite que se reparen los daños en el ADN). Finalmente la senescencia conduce a la muerte celular por un mecanismo diferente a la apoptosis. Las células senescentes en cultivo conservan un número limitado de divisiones celulares que han perdido su capacidad de proliferación habiendo llegado al límite de su vida replicativa.

En relación a los acontecimientos moleculares reguladores o estimuladores del ciclo celular en el liquen plano oral, diversas publicaciones han co- 
municado que las células agredidas desarrollan mecanismos moleculares encaminados a detener el ciclo celular para reparar el ADN, inducir senescencia celular o inducir apoptosis para eliminar las células con ADN severamente lesionada. Sin embargo, otros autores también han demostrado que las células epiteliales en el LPO frecuentemente responden al ataque $\mathrm{T}$ - linfocitario con un incremento de los índices de proliferación celular $(7,8)$. Desde nuestro punto de vista resulta sorprendente e inesperada la concurrencia en el epitelio oral afectado por LP de mecanismos moleculares que pueden detener el ciclo celular, inducir apoptosis y estimular la proliferación celular.

Por otra parte el potencial de transformación maligna del LPO podría estar en relación con los fenómenos contradictorios que acontecen en la regulación del ciclo de las células epiteliales agredidas.

\section{OBJETIVOS}

1. Estudiar la presencia de marcadores de apoptosis.

2. Conocer la importancia cuantitativa de los fenómenos apoptóticos en el LPO.

3. Comprobar la validez de las técnicas de TUNEL y de la peroxidasa-antiperoxidasa para la detección de la muerte celular programada en el LPO.

4. Establecer una hipótesis etiopatogénica sobre la influencia de los mecanismos apoptóticos y de control del ciclo celular en el proceso de malignización del LPO, que sirva de punto de partida para el estudio de este importante evento.

\section{PACIENTES Y MÉTODOS}

Realizamos un estudio en 52 pacientes con LPO vistos en la Universidad Complutense de Madrid siguiendo un proceso de exploración e historia clínica completa así como biopsia.

Los criterios de inclusión fueron:

1. LPO diagnosticado clínicamente con ausencia de displasia y signos de malignización.

2. Bloques de parafina con el grosor suficiente.
3. Disponibilidad de datos clínicos y filiación del paciente.

La muestra fue de 32 pacientes con LPO y como control se utilizó un grupo de 20 personas a las que se les tomaron muestras de mucosa del área retromolar en las cirugías de terceros molares.

Entre las variables demográficas y clínicas se consideró el sexo, la edad, el tipo clínico de la lesión (considerando lesiones reticulares puras y lesiones atrófico-erosivas), el lugar de toma de la biopsia, el tiempo de evolución de la enfermedad ( $<6$ meses, entre 6 y 12 meses, > de 12 meses), los hábitos nocivos como consumo de tabaco y alcohol, el estado emocional del paciente; así mismo se realizó una analítica para evaluar la presencia de VHC y VHB, preguntando sobre otras enfermedades que padeciera y la medicación que tomara para las mismas.

Se realizó una tinción con hematoxilina-eosina de las secciones tisulares tanto de los casos, como de los controles para el estudio histopatológico

En el estudio histopatológico se consideraron las siguientes variables: queratosis (considerándose la posibilidad de que se tratara de ortoqueratosis o paraqueratosis). Presencia de acantosis, granulosis, espongiosis, degeneración vacuolizante de la capa basal del epitelio, cuerpos de Civatte, exocitosis linfocitaria e infiltrado inflamatorio subepitelial en banda.

Para la valoración de estos parámetros se aplicó un método de evaluación semicuantitativa considerándose las categorías: ausencia, leve, moderada, intensa, no valorables. También se observó la morfología de la unión epitelio conectiva considerándose las posibilidades de presentación de una morfología: plana, papilar, no valorable. Finalmente se consideró la presencia o ausencia de despegamientos epiteliales.

Para la valoración de la expresión de los marcadores de apoptosis, Caspasa-3 y Bax, y de la expresión del marcador de detención del ciclo y senescencia p21, se realizó una inmunohistoquímica siguiendo la técnica de peroxidasa-antiperoxidasa (PAP) y utilizando anticuerpos policlonales anti-Caspasa-3 (Pharmingen $\AA)$, anti-p21 y anti-Bax (Santa Cruz $\left.{ }^{\circledR}\right)$. 
Además también se realizó una valoración de la apoptosis empleando la técnica TUNEL con el KIT QIA 33 (Oncogene ${ }^{\circledR}$ ). Se trata de un sistema no isotópico que marca la fragmentación del ADN en los núcleos apoptóticos. Para su aplicación se siguieron específicamente las indicaciones del fabricante.

Para la valoración de los resultados de las técnicas inmunohistoquímicas y de la técnica TUNEL se realizó un recuento celular en cuatro campos de gran aumento consideramos las siguientes categorías en función del \% de positividad: negativo, levemente positivo (<10\%), moderadamente positivo (10 $\leq 25 \%$ ), fuertemente positivo $(25 \leq 50 \%)$. En ningún caso encontramos lesiones con más del $50 \%$ de células positivas para ninguno de los marcadores.

Se realiza un estudio descriptivo de los pacientes y las lesiones. Se observan las asociaciones estadísticas entre casos y controles según los diferentes anticuerpos utilizados. Después se compara el tipo clínico (reticular y atrófico-erosivo) con las diferentes variables descriptivas, clínicas e histológicas. Se comparan las variables inmunohistoquímicas (TUNEL, p21, Caspasa-3 y Bax) con las descriptivas, clínicas e histológicas. Y por último un estudio comparativo entre las distintas variables inmunohistoquímicas.

Se realizó un análisis estadístico calculándose las medias, las desviaciones estándar y los porcentajes de las variables analizadas. Se establecieron asociaciones bivariantes simples empleando diferentes test dependiendo de las variables (dicotómicas, categóricas, ordinales o cuantitativas) los test chi-cuadrado, exacto de Fisher bilateral, Mann-Whitney, T de Student o correlación de Spearman, según interesara en cada comparación. Además se ha calculado la Odds ratio para observar la influencia de las variables caso/control sobre el resto variables.

\section{RESULTADOS}

Las muestras de pacientes con LPO fueron de un $62,5 \%$ de mujeres frente a un 37,5\% de varones (Gráfica 1). Un $8 \%$ y $4 \%$ de los pacientes presentaron marcadores positivos para el VHC y VHB (virus hepatitis $\mathrm{C}$ y B). (Tabla 1 ) El $56,2 \%$ se presentó con formas reticulares puras y el $43,8 \%$ con formas atró-

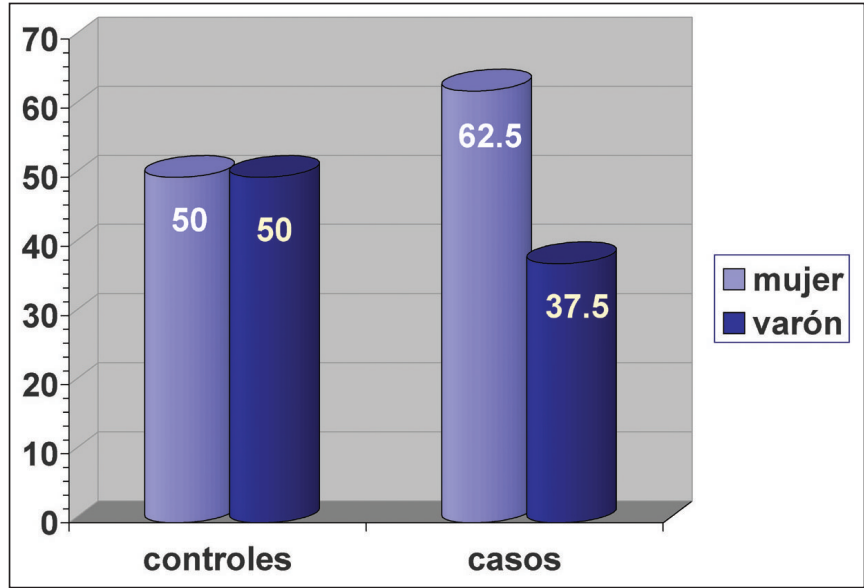

Gráfica 1. Distribución según el sexo de los pacientes con LPO.




fico-erosivas de los que un 46,9\% presentaron síntomas. El 13,3\% de los pacientes tuvieron afectación extraoral (Tabla 2).

La máxima frecuencia de afectación fue en la mucosa yugal $(59,4 \%)$ frente a un $15,6 \%$ en encía y un $15,6 \%$ en mucosa labial. La lengua presentó una frecuencia del 6,3\% (Gráfica 2).

El tiempo de evolución del LPO se puede ver en la tabla 3 siendo menos de 6 meses el 28,1\% y más de 12 meses el 43,8\% de la muestra (Tabla 3 ).

\begin{tabular}{|lrc|}
\hline \multicolumn{3}{|c|}{ TABLA 2. DESCRIPCIÓN DE VARIABLES } \\
CLÍNICAS EN EL GRUPO DE CASOS CON \\
\multicolumn{3}{|c|}{ LP=32) } \\
\hline Variable & n \\
\hline Tipo clínico & & \\
- Reticular & 18 & 56,2 \\
- Atrófico-erosivo & 14 & 43,8 \\
Otras localizaciones intraorales & & \\
- No & 17 & 56,7 \\
- Sí & 13 & 43,3 \\
- No valorado & 2 & \\
Otras localizaciones & & \\
extraoraorales & & \\
- No & 26 & 86,7 \\
- Sí & 4 & 13,3 \\
Síntomas valorado & 2 & \\
- No & & \\
- Sí & 17 & 53,1 \\
\end{tabular}

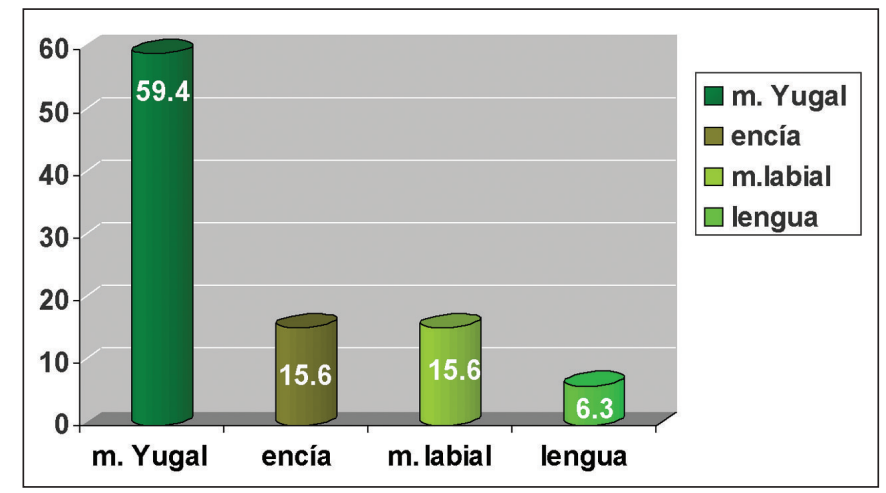

Gráfica 2. Distribución del porcentaje de biopsias según la localización.
En las tinciones Hematoxilina-eosina se observaron en los casos microespongiosis, muerte queratinocítica, cuerpos de Civatte, exocitosis linfocitaria, hiperqueratosis, acantosis e infiltrado inflamatorio. Lo más destacable de las características histopatológicas de nuestra serie es la alta frecuencia de casos con infiltrado inflamatorio intenso $(56,3 \%)$

Lo más interesante son los resultados en cuanto a la expresión de marcadores de apoptosis y de detención del ciclo celular.

Con la técnica de TUNEL en los controles el resultado es negativo en la totalidad de células de la capa suprabasal y de la capa basal. Hay pocas células positivas en el infiltrado. Sin embargo en los casos en la capa basal y suprabasal (sobre todo en el capa espinosa y granulosa) los queratinocitos están teñidos en su mayoría, siendo en la capa basal de leve a moderada $(+,++)$ y en la suprabasal de leve intensidad. Esto indicará que la apoptosis es un fenómeno celular que forma parte de los mecanismos moleculares que se desarrollan en el LPO y que las células de la capa basal constituyen la diana de la agresión T-linfocitaria. La estadística nos concluye que hay diferencia estadísticamente significativa entre casos y controles, siendo la expresión significativamente mayor en la basal $(p=0,004)($ Tabla 4$)$.

Sin embargo de acuerdo con Dekker, Bloor, Neppelberg y Tobon (9-12) nosotros encontramos que los fenómenos apoptóticos son de poca impor-

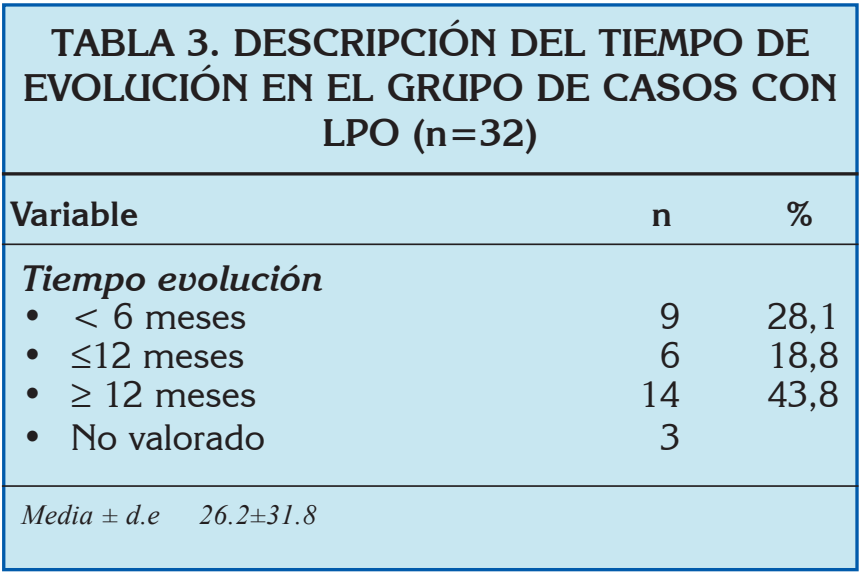




\begin{tabular}{|c|c|c|}
\hline $\begin{array}{r}\text { TABLA } 4 . \\
\text { VARIABLES HIS } \\
\text { EN LOS CA }\end{array}$ & $\begin{array}{l}E \mathrm{LA} \\
\mathrm{EP} \\
\mathrm{N}=3\end{array}$ & LIO \\
\hline Variable & $\mathrm{n}$ & $\%$ \\
\hline Queratosis & & \\
\hline - No & 4 & 13,8 \\
\hline - Paraqueratosis & 9 & 31,0 \\
\hline - Ortoqueratosis & 16 & 55,2 \\
\hline - No valorado & 3 & \\
\hline Acantosis & & \\
\hline - - & 5 & 18,5 \\
\hline - + & 11 & 40,7 \\
\hline - ++ & 9 & 33,3 \\
\hline - +++ & 2 & 7,4 \\
\hline - No valorado & 5 & \\
\hline Granulosis & & \\
\hline - - & 2 & 7,4 \\
\hline - + & 15 & 55,6 \\
\hline - ++ & 9 & 33,3 \\
\hline - +++ & 1 & 3,7 \\
\hline - No valorado & 5 & \\
\hline Espongiosis & & \\
\hline - - & 20 & 71,4 \\
\hline - + & 7 & 25,0 \\
\hline - ++ & 1 & 3,6 \\
\hline - $\quad+++$ & 0 & 0,0 \\
\hline - No valorado & 4 & \\
\hline Vacuolización & & \\
\hline - - & 0 & 0,0 \\
\hline - + & 21 & 72,4 \\
\hline - ++ & 5 & 17,2 \\
\hline - +++ & 3 & 10,3 \\
\hline - No valorado & 3 & \\
\hline Cuerpos de Civat & & \\
\hline - - & 2 & 7,1 \\
\hline - $\quad+$ & 13 & 46,6 \\
\hline - ++ & 7 & 25,0 \\
\hline - +++ & 6 & 21,4 \\
\hline - No valorado & 4 & \\
\hline Exocitosis linfocit & & \\
\hline$\cdot-$ & 2 & 6,7 \\
\hline - $\quad+$ & 14 & 46,7 \\
\hline - ++ & 5 & 16,7 \\
\hline - +++ & 9 & 30,0 \\
\hline - No valorado & 2 & \\
\hline
\end{tabular}

tancia cuantitativa en el LPO. Así en la capa basal, los resultados de la técnica TUNEL demostraron que el $13,8 \%$ de nuestros casos fueron negativos y el $62,1 \%$ expresaron signos de apoptosis en menos del $10 \%$ de las células basales (Tabla 5).

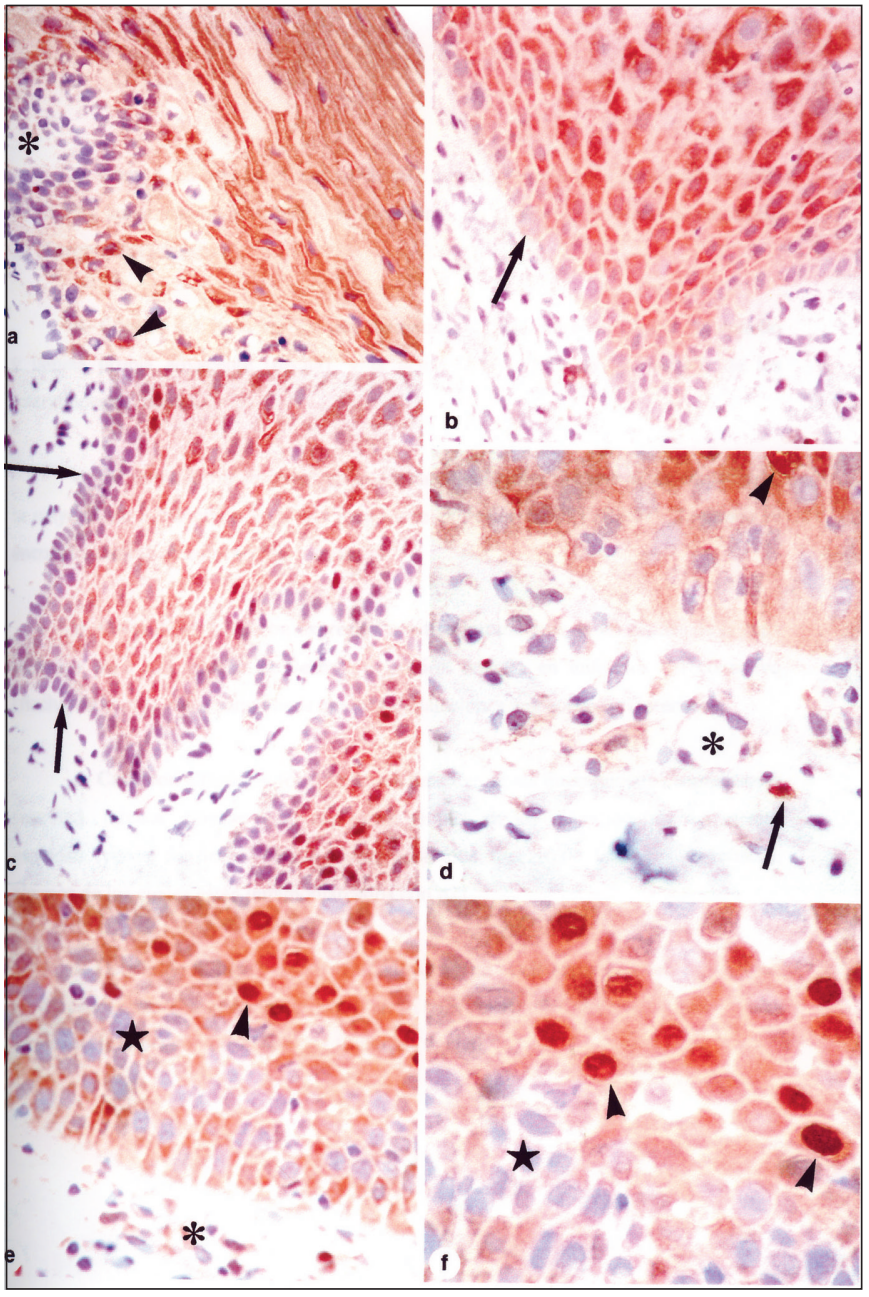

Figura 2. a. Control sin expresión nuclear de p21 en los queratinocitos parabasales (punta de flecha), aunque se observa teñido el citoplasma. En el corion se observa la migración de micropapilas (asterisco).

b. Control donde se aprecia una delimitación nítida entre capa basal del epitelio y lámina propia adyacente (flecha), y la negatividad a p21 de los núcleos de los queratinocitos basales.

c. En este control se observan queratinocitos basales dispuestos en empalizada, la mayoría sin inmunomarcaje o con muy tenue expresión de p21 (flecha). Tampoco se encuentra expresión en el corion subyacente.

d. Corte de un LPO con expresión positiva intranuclear de las células de la cpa espinosa para p21 (punta de flecha). Expresión de p21 en linfocitos del conectivo (flecha). Algunos vasos sanguíneos muestran dilataciones angiectásicas (asterisco).

e. Caso con alteración focal de la maduración de los queratinocitos suprabasales (estrella). Intensa, aunque focal expresión de p21 en los queratinocitos del estrato espinoso (punta de flecha). Se aprecian infiltrados focales de linfocitos (asterisco), algunos de los cuales están inmunomarcados con p21.

f. Detalle de la figura anterior, donde se aprecian las áreas de microespongiosis en las células suprabasales que no expresan p21 (estrella), mientras que en otras células se aprecia la expresión positiva a p21 (punta de flecha). 


\section{TABLA 5. ASOCIACIÓN ENTRE LOS CASOS DE LPO Y LOS CONTROLES CON LA TÉCNICA INMUNOHISTOQUIIMICA DE TÚNEL}

\begin{tabular}{|c|c|c|c|c|c|c|}
\hline \multirow[t]{2}{*}{ Variable } & \multicolumn{2}{|c|}{ Controles $(n=20)$} & \multicolumn{2}{|c|}{ Casos $(n=32)$} & \multirow[t]{2}{*}{ Comparación } & \multirow[t]{2}{*}{ Odds Ratio } \\
\hline & $\mathbf{n}$ & $\%$ & & $\%$ & & \\
\hline $\begin{array}{l}\text { Capa suprabasal TUNEL } \\
\cdot- \\
\cdot+ \\
\cdot++ \\
\cdot+++ \\
\cdot+\text { No valorado }\end{array}$ & $\begin{array}{r}17 \\
3 \\
0 \\
0 \\
0\end{array}$ & $\begin{array}{r}85,0 \\
15,0 \\
0,0 \\
0,0\end{array}$ & $\begin{array}{l}7 \\
2 \\
1 \\
1 \\
0 \\
3\end{array}$ & $\begin{array}{r}24,1 \\
72,4 \\
3,4 \\
0,0\end{array}$ & $U=112,0, p<0,001^{a}$ & $\mathrm{OR}=17,81^{\mathrm{c}}$ \\
\hline $\begin{array}{l}\text { Capa basal TUNEL } \\
\bullet- \\
\cdot+ \\
\cdot++ \\
\cdot+++ \\
\cdot+\text { No valorado }\end{array}$ & $\begin{array}{r}17 \\
3 \\
0 \\
0 \\
0\end{array}$ & $\begin{array}{r}85,0 \\
15,0 \\
0,0 \\
0,0\end{array}$ & $\begin{array}{l}4 \\
1 \\
8 \\
6 \\
1 \\
3\end{array}$ & $\begin{array}{r}13,8 \\
62,1 \\
20,7 \\
3,4\end{array}$ & $U=73,0, p<0,001^{\mathrm{a}}$ & $\mathrm{OR}=25,42^{\mathrm{c}}$ \\
\hline $\begin{array}{l}\text { Infiltrado TUNEL } \\
:- \\
:+ \\
\bullet++ \\
:+++ \\
- \text { No valorado }\end{array}$ & $\begin{array}{r}18 \\
2 \\
0 \\
0 \\
0\end{array}$ & $\begin{array}{r}90,0 \\
10,0 \\
0,0 \\
0,0\end{array}$ & $\begin{array}{l}1 \\
0 \\
1 \\
9 \\
0 \\
0 \\
3\end{array}$ & $\begin{array}{r}34,5 \\
65,5 \\
0,0 \\
0,0\end{array}$ & $\chi_{\mathrm{c}}^{2}=12,72, p<0,001^{\mathrm{b}}$ & $\mathrm{OR}=17,10^{c}$ \\
\hline
\end{tabular}

En cuanto al p21 vemos diferencias significativas entre casos y control (Tabla 6). En los controles Figura $2(a, b, c)$ es negativo en la totalidad de las células de la capa suprabasal y de la basal observándose escasas células positivas en el infiltrado. En el epitelio de los casos de LPO en su capa basal y suprabasal (en especial en la capa espinosa y en algunas de la granulosa) los queratinocitos están teñidos en su mayoría, siendo en su capa basal casi todos de leve intensidad y en las capas suprabasales de leves moderada $(+,++)$. En el infiltrado inflamatorio en un $80 \%$ son leves $(+)$ (Figura 2$)$.

Estadísticamente se puede afirmar que los casos de LPO presentan una mayor posibilidad de ser positivos en la capa suprabasal con la técnica de inmunohistoquímica para p21 que los controles $(p<0,001)$. La odds ratio en esta variable no es valorable. En la capa basal los casos presentan una mayor posibilidad de ser positivos que los controles ( $p<0,001)$. La odds ratio tampoco es valorable. En cuanto al infiltrado los casos tienen mas posibilidades de ser positivos que los controles $(\mathrm{p}<0,001)$. La odds ratio para esta variable es 17,33 (Tabla 6).

En relación con la técnica anticaspasa 3 en los controles, hemos encontrado negatividad en todas las capas de la muestra. En las muestras de LPO el número de células positivas para caspasas 3 en las capas suprabasales es la mitad de la muestra, en la basal casi todas son positivas y en el infiltrado más de la mitad (Figura 3).

Hay diferencia significativa entre casos y controles en todas las capas. La función de positividad de caspasa 3 es estadísticamente superior en la capa 




Figura 3. a. Control en el que se puede observar la negatividad celular a Caspasa-3 tanto en el epitelio como en el corion. b. Moderada inmunoexpresión de Caspasa-3 en el citoplasma de algunos queratinocitos (flecha) de un caso. En el corion se observan microvesículas (asterisco) asociadas a infiltrados de células linfoides y a dilataciones angiectásicas. c. Expresión bien definida de positividad a Caspasa-3 en forma de punteado marrón en los citoplasmas celulares en un liquen plano oral. $\boldsymbol{d}$. Detalle de la figura anterior en la que se observa más nítidamente la expresión granular intracitoplasmática de Caspasa-3 (flecha). e. Caso con microespongiosis en la capa basal del epitelio (asterisco) y células inflamatorias de Caspasa-3 positivas en áreas de degeneración inflamatoria del corion (flechas negras). f. Expresión positiva para la Caspasa-3 en los citoplasmas celulares (flechas negras) y edema (asterisco).

basal en comparación con la suprabasal $(p=0,052)$. (Tabla 7).

Solo el estudio de Tobon (4) presentó unos datos similares a los nuestros. Según Harmon, Collins, GraslKraupp, Druilhe y Marshaman (13-16) se trataba de un marcador altamente específico y sensiblemente de apoptosis que permite diferenciar con una alta sensibilidad y especificidad a la técnica de TUNEL, la muerte por apoptosis de la muerte por necrosis.
Con el Bax nuestros resultados difieren de los encontrados en el resto de los marcadores de apoptosis. Tanto en controles como en los casos las células se tiñen pero de intensidad leve a moderada y en la capa basal suprabasal así como en el infiltrado la función es de moderada a intensa (Tabla 8) (Figura 4).

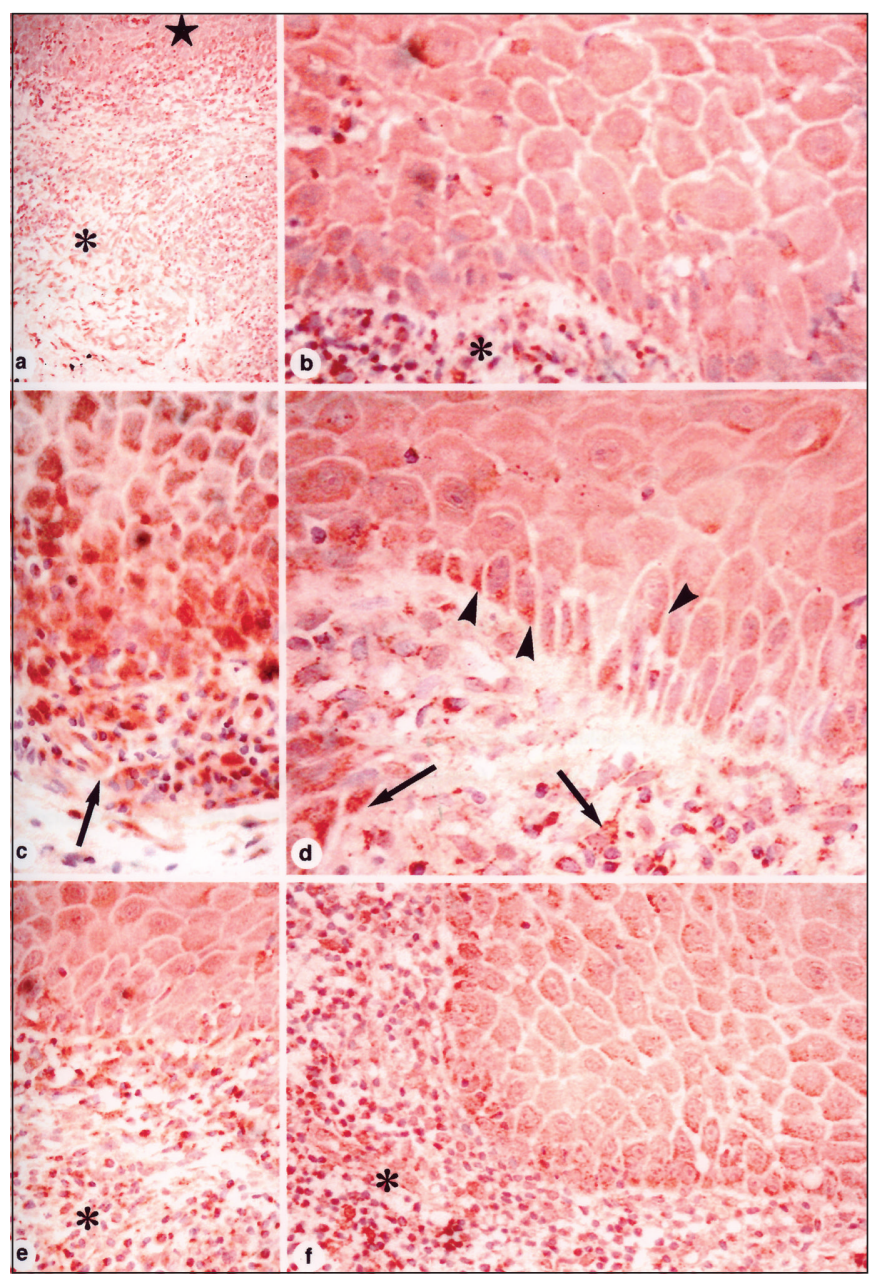

Figura 4. a. Liquen plano oral donde se observa la positiva a Bax en el epitelio (estrella) y también su positividad en el conectivo (asterisco). b. Caso en el que se puede apreciar la positividad a Bax de los citoplasmas celulares en el conectivo (asterisco). c. LPO con abundante número de queratinocitos teñidos en el epitelio e intenso infiltrado inflamatorio en la limitante epitelio-conectiva (flecha). d. Corte correspondiente a una caso con intensa expresión inmunocitoplásmica granular (puntas de flecha) de Bax en queratinocitos basales y suprabasales. A su vez, se da expresión de Bax en las células inflamatorias del conectivo (flechas). e. Corresponde a un caso en el que se aprecia la positividad a Bax a nivel corion (asterisco). f. En este LPO se ve una masiva infiltración de linfocitos y otras células inmunocomponentes en la lámina propia (asterisco). También se observa una intensa tinción celular en la capa basal. 


\begin{tabular}{|c|c|c|c|c|c|c|}
\hline TABLA 6. ASOCI & YTl & $\begin{array}{l}\text { S CAS } \\
\text { NOHIs }\end{array}$ & & $\begin{array}{ll}\mathrm{YL} \\
\mathrm{CO}\end{array}$ & 21 & ECTICA \\
\hline Variable & Con & $s(n=20)$ & & $n=32)$ & Comparación & Odds Ratio \\
\hline & n & $\%$ & & $\%$ & & \\
\hline $\begin{array}{l}\text { Capa suprabasal p21 } \\
:- \\
\cdot+ \\
\cdot++ \\
\cdot+++ \\
\cdot+\text { No valorado }\end{array}$ & $\begin{array}{r}20 \\
0 \\
0 \\
0 \\
0\end{array}$ & $\begin{array}{r}100,0 \\
0,0 \\
0,0 \\
0,0\end{array}$ & $\begin{array}{l}9 \\
9 \\
7 \\
3 \\
4\end{array}$ & $\begin{array}{l}32,1 \\
32,1 \\
25,0 \\
10,7\end{array}$ & $U=90,0, p<0,001^{a}$ & $\begin{array}{c}\mathrm{OR}=\mathrm{No} \\
\text { valorable }\end{array}$ \\
\hline $\begin{array}{l}\text { Capa basal p21 } \\
\bullet- \\
\cdot+ \\
\cdot++ \\
\cdot++ \\
\cdot+\text { No valorado }\end{array}$ & $\begin{array}{r}20 \\
0 \\
0 \\
0\end{array}$ & $\begin{array}{r}100,0 \\
0,0 \\
0,0 \\
0,0\end{array}$ & $\begin{array}{l}9 \\
1 \\
6 \\
3 \\
0 \\
4\end{array}$ & $\begin{array}{r}32,1 \\
57,1 \\
10,7 \\
0,0\end{array}$ & $U=90,0, p<0,001^{a}$ & $\begin{array}{c}\mathrm{OR}=\mathrm{No} \\
\text { valorable }^{\mathrm{b}}\end{array}$ \\
\hline $\begin{array}{l}\text { Infiltrado p21 } \\
\bullet- \\
\cdot+ \\
\cdot++ \\
\cdot+++ \\
\cdot+\text { No valorado }\end{array}$ & $\begin{array}{r}13 \\
7 \\
0 \\
0 \\
0\end{array}$ & $\begin{array}{r}65,0 \\
35,0 \\
0,0 \\
0,0\end{array}$ & $\begin{array}{l}3 \\
2 \\
5 \\
3 \\
0\end{array}$ & $\begin{array}{r}32,1 \\
57,1 \\
10,7 \\
0,0\end{array}$ & $U=128,0, p<0,001^{a}$ & $\mathrm{OR}=17,33^{\mathrm{b}}$ \\
\hline
\end{tabular}

\section{TABLA 7. ASOCIACIÓN ENTRE LOS CASOS DE LPO Y LOS CONTROLES CON LA TÉCNICA INMUNOHISTOQUÍMICA CON CASPASA-3}

\begin{tabular}{|c|c|c|c|c|c|c|}
\hline \multirow[t]{2}{*}{ Variable } & \multicolumn{2}{|c|}{ Controles $(n=20)$} & \multicolumn{2}{|c|}{ Casos $(n=32)$} & \multirow[t]{2}{*}{ Comparación } & \multirow[t]{2}{*}{ Odds Ratio } \\
\hline & & $\%$ & & $\%$ & & \\
\hline $\begin{array}{l}\text { Capa suprabasal Caspasa-3 } \\
:- \\
\cdot+ \\
\bullet++ \\
\cdot+++ \\
- \text { No valorado }\end{array}$ & $\begin{array}{r}20 \\
0 \\
0 \\
0 \\
0\end{array}$ & $\begin{array}{r}100,0 \\
0,0 \\
0,0 \\
0,0\end{array}$ & $\begin{array}{l}1 \\
4 \\
1 \\
4 \\
0 \\
0 \\
4\end{array}$ & $\begin{array}{r}50,0 \\
50,0 \\
0,0 \\
0,0\end{array}$ & $\chi_{c}^{2}=11,80, p<0,001^{a}$ & $\begin{array}{c}\mathrm{OR}=\mathrm{No} \\
\text { valorable }^{\mathrm{c}}\end{array}$ \\
\hline $\begin{array}{l}\text { Capa basal Caspasa-3 } \\
\bullet-+ \\
:++ \\
:++ \\
:+++ \\
- \text { No valorado }\end{array}$ & $\begin{array}{r}20 \\
0 \\
0 \\
0 \\
0\end{array}$ & $\begin{array}{r}100,0 \\
0,0 \\
0,0 \\
0,0\end{array}$ & $\begin{array}{l}7 \\
2 \\
1 \\
0 \\
0 \\
4\end{array}$ & $\begin{array}{r}25,0 \\
75,0 \\
0,0 \\
0,0\end{array}$ & $\chi_{c}^{2}=23,71, p<0,001^{a}$ & $\begin{array}{c}\mathrm{OR}=\mathrm{No} \\
\text { valorable }^{\mathrm{c}}\end{array}$ \\
\hline \begin{tabular}{|l} 
Infiltrado Caspasa-3 \\
$:-$ \\
$\bullet+$ \\
$\bullet++$ \\
$:+++$ \\
$\cdot+$ No valorado
\end{tabular} & $\begin{array}{r}20 \\
0 \\
0 \\
0 \\
0\end{array}$ & $\begin{array}{r}100,0 \\
0,0 \\
0,0 \\
0,0\end{array}$ & $\begin{array}{l}1 \\
2 \\
1 \\
4 \\
2 \\
0 \\
4\end{array}$ & $\begin{array}{r}42,9 \\
50,0 \\
7,1 \\
0,0\end{array}$ & $U=120,0, p<0,001^{b}$ & $\begin{array}{c}\mathrm{OR}=\mathrm{No} \\
\text { valorable }\end{array}$ \\
\hline
\end{tabular}




\begin{tabular}{|c|c|c|c|c|c|c|}
\hline \multirow[t]{2}{*}{ Variable } & \multicolumn{2}{|c|}{ Controles $(n=20)$} & \multicolumn{2}{|c|}{ Casos $(n=32)$} & \multirow[t]{2}{*}{ Comparación } & \multirow[t]{2}{*}{ Odds Ratio } \\
\hline & $\mathbf{n}$ & $\%$ & & $\%$ & & \\
\hline $\begin{array}{l}\text { Capa suprabasal Bax } \\
:- \\
\cdot++ \\
\cdot++ \\
\cdot+++ \\
- \text { No valorado }\end{array}$ & $\begin{array}{r}0 \\
6 \\
8 \\
2 \\
16\end{array}$ & $\begin{array}{r}0,0 \\
37,5 \\
50,0 \\
12,5\end{array}$ & $\begin{array}{l}0 \\
3 \\
8 \\
1 \\
1 \\
1 \\
0\end{array}$ & $\begin{array}{r}0,0 \\
13,6 \\
36,4 \\
50,0\end{array}$ & $U^{a}=98,0, p<0,014^{a}$ & $\begin{array}{c}\mathrm{OR}=\mathrm{No} \\
\text { valorable }\end{array}$ \\
\hline $\begin{array}{l}\text { Capa basal Bax } \\
:-+ \\
\bullet++ \\
\bullet++ \\
:+++ \\
\cdot \text { No valorado }\end{array}$ & $\begin{array}{r}0 \\
8 \\
5 \\
3 \\
16\end{array}$ & $\begin{array}{r}0,0 \\
50,0 \\
31,3 \\
18,8\end{array}$ & $\begin{array}{l}0 \\
6 \\
6 \\
1 \\
0 \\
1 \\
0\end{array}$ & $\begin{array}{r}0,0 \\
27,3 \\
27,3 \\
45,5\end{array}$ & $U=120,0, p<0,079^{a}$ & $\begin{array}{c}\mathrm{OR}=\mathrm{No} \\
\text { valorable }^{\mathrm{b}}\end{array}$ \\
\hline $\begin{array}{l}\text { Infiltrado Bax } \\
\bullet-+ \\
\bullet+ \\
\cdot++ \\
\cdot+++ \\
\cdot+\text { No valorado }\end{array}$ & $\begin{array}{r}2 \\
10 \\
4 \\
0 \\
16\end{array}$ & $\begin{array}{r}12,5 \\
62,5 \\
25,0 \\
0,0\end{array}$ & $\begin{array}{l}1 \\
1 \\
5 \\
1 \\
3 \\
1 \\
2\end{array}$ & $\begin{array}{r}5,0 \\
5,0 \\
25,0 \\
65,0\end{array}$ & $U=34,0, p<0,001^{a}$ & $\mathrm{OR}=2,71^{\mathrm{b}}$ \\
\hline
\end{tabular}

A la pregunta de cual es la razón de los altos niveles de apoptosis encontrados con Bax en tejido sano (dado que se debería esperar poca frecuencia) no vemos una explicación plausible, lo que podría solo significar la poca utilidad del Bax como marcador específico de apoptosis. La muerte apoptótica masiva en las células basales del LPO, será muy grave para el epitelio ya que esta podrá perder su capacidad de regeneración sucumbiendo al ataque de linfocitos $\mathrm{T}$ en un proceso que se podría denominar claudicación epitelial.

El establecimiento de mecanismos moleculares celulares de resistencia a la agresión que no impliquen la muerte celular apoptótica podrían ser biológicamente más prudente, ya que permitirían la recuperación potencial de las células atacadas y el mantenimiento de la cinética celular y la posibilidad de reconstrucción de la estructura epitelial.
Por estas funciones, la capa basal del epitelio oral, la única capa en la que las células tienen capacidad replicativa, no debe expresar p21, tal y como lo han reconocido otros autores.

Parecería razonable proponer la hipótesis que las alteraciones moleculares relacionadas con el control celular del ciclo pueden producir un substrato epitelial favorable a la evolución hacia la malignidad. Se requieren más estudios para demostrar taxativamente esta hipótesis.

\section{BIBLIOGRAFÍA}

1. Bloor Bk, Malik Fk, Odell EE, Morgan PR. Quantitative assessment of apoptosis in oral lichen planus. Oral Surg Oral Med Oral Pathol Radios Endod 1999;88:187-95 
2. Dekker NP, Lozada-Nür F, Laguenam LA, MacPhail LA, Bloom CY, Regezi JA. Apoptosisassociated markers in oral lichen planus. J Oral Pathol Med 1997;26:170-5

3. Neppelberg E, Johannesen Ac, Jonsson R. Apoptosis in oral lichen planus. Eur J Oral Scir 2001; 109:361-4

4. Tobón SI, Villegas FA, Ruiz SM, Vieco B, Restrepo M, Londoño ML. Expresión of caspase-3 and structural changes associated with apoptotic cell death of keratinocytes in oral lichen planus. Oral and Maxillofacial Patology 2004;10:173-8

5. Druilhe A, Benoit W, Tsicopulos A, Lapa JR, TillieLeoblond I, tonel A, Pretolani M. Apoptosis, proliferation, and expression of bcl-2, Fas and Fas-ligand in bronchial biopsies from asthmatics. Am J Respir Cell Moll boil 1998;19:747-57

6. Grasl-Kraupp B, Ruttakay-nedecky B, Koudelka H, Bukowska K, Bursch W, Schulle-Hermann R. In situ detection of fragmented DNA (TUNEL assay) fails to discriminate among apoptosis, necrosis and autolytic cell death: a cautionary note. Histopathol 1995;21:1465-8

7. Wong S, Chan J, Lee K, Hsiao W. Differential expression of p16/p21/p27 and Cyclin D1/D3, and their relationships to cell proliferation, apoptosis, and tumour progression in invasive ductal carcinoma of the breast. J Pathol 2001;194:35-42.

8. Arends M.J, Morris R.G and Wyllie A.H. Apoptosis; The role of the endonuclease. American Journal of Pathology 1990; vol 136:593-608.

9. Dekker NP, Lozada-Nür F, Laguenam LA, MacPhail LA, Bloom CY, Regezi JA. Apoptosisassociated markers in oral lichen planus. J Oral Pathol Med 1997;26:170-5.

10. Bloor BK, Malik FK, Odell EW, Morgan PR. Quantitative assessment of apoptosis in oral lichen planus. Oral Surg Oral Med Oral Pathol Radiol Endod 1999;88:187-95.
11. Neppelberg E, Johannesen AC, Jonsson R. Apoptosis in oral lichen planus. Eur J Oral Sci 2001; 109:361-4.

12. Tobón SI, Villegas FA, Ruiz SM, Vieco B, Restrepo $M$, Londoño ML. Expression of caspase-3 and structural changes associated with apoptotic cell death of keratinocytes in oral lichen planus. Oral and Maxillofacial Pathology 2004;10:173-8

13. Harmon BV, Corder AM, Collins RJ, Gobe GC, Allen J, Allan DJ, Kerr JF. Cell death induced in a murine mastocytoma by $42-47$ degrees $C$ heating in vitro: evidence that the form of death changes from apoptosis to necrosis above a critical heat load. Int J Radiat Biol 1990;58:845-58.

14. Collins Harmon BV, Gobe GC, Kerr JFR. Internucleosomal DNA cleavage should not be the sole criterion for identifying apoptosis. Int $\mathrm{J}$ Radiat Biol 1992;61:451-3.

15. Grasl-Kraupp B, Ruttakay-Nedecky B, Koudelka H, Bukowska K, Bursch W, Schulte-Hermann R. In situ detection of fragmented DNA (TUNEL assay) fails to discriminate among apoptosis, necrosis and autolytic cell death: a cautionary note. Histopathol 1995;21:1465-8.

16. Druilhe A, Benoit W, Tsicopoulos A, Lapa JR, Tillie-Leoblond I, Tonel A, Pretolani M. Apoptosis, proliferation, and expression of bcl-2, Fas and Fas-ligand in bronchial biopses from asthmatics. Am J Respir Cell Moll Biol 1998;19:747-57.

\section{CORRESPONDENCIA}

Prof. Antonio Bascones

Departamento de Medicina y Cirugía Bucofacial

Facultad de Odontología.

Universidad Complutense

Plaza Ramón y Cajal, s/n

28040 Madrid, Spain.

E-mail: antbasco@arrakis.es

Teléfono: +34913942019

Fax: +34 915338064 
